\title{
Detection of plasma exosomal miRNA-205 as a biomarker for early diagnosis and an adjuvant indicator of ovarian cancer staging
}

Zehua Zhu' ${ }^{1}$, Zhaojun Chen ${ }^{2}$, Mingxing Wang ${ }^{1}$, Min Zhang ${ }^{3}$, Yiwen Chen ${ }^{2}$, Xiao Yang ${ }^{2}$, Changjun Zhou ${ }^{2}$, Yuhua Liu², Liquan Hong ${ }^{2}$ and Lahong Zhang ${ }^{2^{*}}$ (D

\begin{abstract}
Background: Ovarian cancer $(\mathrm{OC})$ is one of the serious threats to the health of women worldwide, and accurate biomarkers are urgently demanded for early diagnosis of OC. We have previously confirmed that miR-205 promotes the invasion and metastasis of OC cells by inhibiting the expression of the tumor suppressor gene TCF21. In this study, we used liquid biopsy technology to detect the expression levels of the four genes, miR-205, CA125, HE4 and TCF21, in the exosomes of plasma of OC patients. Combined with analysis of clinicopathological parameters of OC patients, we aimed to provide efficient and non-invasive laboratory biomarkers for early diagnosis of OC.

Methods: 36 OC patients who were diagnosed in local hospitals from September 2020 to July 2021 were selected as OC group, 31 cases of surgically diagnosed with ovarian benign lesions were selected as benign group, and 32 healthy people who underwent physical examination during the same period were selected as a control group. We employed transmission electron microscope (TEM), Western blotting (WB), and nanoparticle tracking analysis (NTA) to identify biomarkers in the exosomes extracted from plasma of the three groups. The RNA levels of miR-205, CA125, HE4 and TCF21 genes in plasma exosomes were detected by real-time quantitative PCR (qRT-PCR) method. We used clinical pathological parameters and the Receiver Operating Characteristic (ROC) curves to evaluate the diagnostic efficacy for the genes detected in plasma exosomes.
\end{abstract}

Results: We found that the expression level of miR-205 in plasma exosomes of the OC group was significantly higher than that of the benign and control groups $(P<0.05)$, and the level of miR-205 was elevated during the III-IV periods of OC and lymph node metastasis.

Conclusion: The level of miR-205 in plasma exosomes is a valuable tumor biomarker to improve OC diagnosis.

Keywords: Ovarian cancer, Exosome, miRNA-205, Diagnosis, Biomarker

*Correspondence: zjhzzlh2007@163.com

2 The Clinical laboratory of The Affiliated Hospital of Hangzhou Normal University, Hangzhou 310015, China

Full list of author information is available at the end of the article

\section{Background}

Ovarian cancer $(\mathrm{OC})$ is one of the most common gynecological malignancies in the female reproductive system with the leading fatality rate, posing a serious threat to the health of women worldwide [1]. Due to the lack of early clinical manifestations and effective screening methods, $70-75 \%$ of the OC patients were already at the original author(s) and the source, provide a link to the Creative Commons licence, and indicate if changes were made. The images or other third party material in this article are included in the article's Creative Commons licence, unless indicated otherwise in a credit line to the material. If material is not included in the article's Creative Commons licence and your intended use is not permitted by statutory regulation or exceeds the permitted use, you will need to obtain permission directly from the copyright holder. To view a copy of this licence, visit http://creativecommons.org/licenses/by/4.0/. The Creative Commons Public Domain Dedication waiver (http://creativeco mmons.org/publicdomain/zero/1.0/) applies to the data made available in this article, unless otherwise stated in a credit line to the data. 
advanced stage at the time of diagnosis and therefore missed the best treatment opportunity [2]. Thus, OC is called the "silent killer" and there is an urgent need for more efficient, non-invasive, and early screening methods to improve the diagnosis of OC patients.

Liquid biopsy technology represented by detection of biomarkers in extracted exosomes has been reported as a potential tool to solve this problem [3]. Exosomes are tiny vesicles secreted by cells with an unique lipid bilayer membrane structure which well protects the inside contents, such as mRNA and miRNA, from degradation [4]. Therefore, exosomes are much more stable than other biomarkers presented in plasma. In the early stages of tumors, cancer cells secrete a large quantity of exosomes, reaching a level of $10^{12} / \mathrm{mL}$ of blood, which makes the quantitative detection of exosomes easier and possible as a new tumor marker [5]. Our previously published studies found that miR-205 is up-regulated in OC tissues. In this study, we mechanistically revealed that miR-205 promoted the invasion and metastasis of OC cells by inhibiting expression of the tumor suppressor gene TCF21 [6], suggesting that miR-205 has the potential as a novel biomarker for OC diagnosis. Currently, transvaginal ultrasound combined with detection of CA125 and HE4 proteins is mainly used for ovarian cancer screening and diagnosis [7]. However, the detection of CA125 and HE4 have their own limitations in early diagnosis of ovarian cancer, which significantly limits their use in clinical practice [8]. We used the exosomal liquid biopsy technology to detect the expression levels of the four genes, miR205, CA125, HE4 and TCF21 in the plasma exosomes of OC patients. Together with analysis of the clinicopathological parameters of OC patients, we explored the diagnostic value and clinical significance of the four genes in plasma exosomes for OC.

\section{Methods}

\section{Study populations}

A total of 99 female subjects were included in the study, including 36 in the OC group, 31 in the benign group, and 32 in the control group. In the OC group, of the 36 ovarian cancer patients, who were admitted to Zhejiang Cancer Hospital from September 2020 to July 2021, 32 had high-grade serous adenocarcinomas, 3 had endometrioid adenocarcinomas, and 1 had clear cell carcinoma. The inclusion criteria were as follows: (1) meeting the relevant diagnostic criteria for $\mathrm{OC}$, and the histological type and the International Union of Obstetrics and Gynecology (FIGO) staging were confirmed by postoperative pathological examination [9]; (2) none of them received any anti-tumor treatments before drawing blood; (3) complete medical records; (4) without other malignant diseases. The benign group had 31 patients with benign ovarian lesions, including 7 serous cystadenoma, 7 endometriotic cyst, 5 mature teratoma, 3 cases of mucinous cystadenoma, 9 cases of simple ovarian cyst. They were surgically diagnosed in the Affiliated Hospital of Hangzhou Normal University during the same period. The control group consisted of 32 healthy women who participated in the physical examination in this hospital during the same period, and excluded for conditions of hypertension, diabetes and diseases or dysfunctions of organs such as heart, liver and kidney. They had no histories of malignant tumors and no dysfunction of important organs.

The OC group had ages ranged from 27 to 90 years with an average of $57 \pm 13$, the benign group was ranged from 24 to 73 years old with an average of $49 \pm 13$, and the control group had ages of 27-77 years old with an average of $51 \pm 14$. There were no significant differences in ages between the three groups $(P>0.05)$. We obtained the informed consent from all individuals, and the study was approved by the hospital ethics committee (Ethics No. 2019 (Len 02)-HS-03).

\section{Plasma collection}

All the subjects without taking foods were drawn 4-6 mL of venous blood anticoagulated with EDTA in the early morning. The blood samples were centrifuged at 3, $500 \mathrm{rpm}$ for $10 \mathrm{~min}$ at $4^{\circ} \mathrm{C}$ to obtain plasma, which were divided into 2 tubes, one stored at $-80^{\circ} \mathrm{C}$ and another one was immediately detected for protein concentrations of CA125 and HE4. If the samples were not detected within $8 \mathrm{~h}$, they were be stored at $4^{\circ} \mathrm{C}$, followed by detection within $24 \mathrm{~h}$.

\section{Detection of plasma protein CA125 and HE4}

The protein concentration of CA125 in plasma was assessed using a Chemiluminescence Immunoassay Analyzer (Abbott ARCHITECT i2000SR, USA), and the HE4 protein concentration using an Electrochemiluminescence Immunoassay Analyzer (Roche Cobas E602, Germany), following the manufacturer's protocols.

\section{Exosome isolation}

Exosomes were isolated from plasma using Total Exosome Isolation Reagent (\#4484450; Invitrogen, USA), according to the manufacturer's instructions. Briefly, the sample was thawed at room temperature, centrifuged first at $2000 \mathrm{~g}$ for $20 \mathrm{~min}$ and next at $10,000 \mathrm{~g}$ for $20 \mathrm{~min}$ to completely remove the cells and debris. Next, $400 \mu \mathrm{L}$ of the plasma supernatant were mixed with $120 \mu \mathrm{L}$ of $1 \times$ phosphate buffered saline (PBS, pH7.4), followed by incubation at $4{ }^{\circ} \mathrm{C}$ for $30 \mathrm{~min}$ and centrifugation at $10,000 \mathrm{~g}$ for $5 \mathrm{~min}$ to obtain pellets as exosomes. The exosome samples were stored at $-80^{\circ} \mathrm{C}$ for further analysis. 


\section{Transmission electron microscopy (TEM)}

Exosomal pellets were resuspend in $1 \times \mathrm{PBS}$, and the suspension was dropped onto the carbon-coated copper mesh for 2 min. After excess liquid was removed, a filter paper was used to drain the grid. Negative staining using phosphotungstic acid was performed for $5 \mathrm{~min}$. The grid was then dried at room temperature, followed by observation at a JEOL-1230 TEM at an acceleration voltage of $100 \mathrm{kV}$.

\section{Nanoparticle tracking analysis (NTA)}

The size and concentration of the exosomes were determined using NTA (VivaCell Biosceinces) on ZetaView PMX 110 (Particle Metrix, Meerbusch, Germany) and the software ZetaView 8.04.02. Briefly, the ZetaView system was calibrated with $110 \mathrm{~nm}$ polystyrene particles, then the separated exosome sample was properly diluted with $1 \times$ PBS buffer to measure particle size and concentration. Data were recorded and analyzed using NTA measurements at a room temperature.

\section{Western blotting analyses (WB)}

The exosome samples were treated with lysis buffer to obtain total exosomal protein, which was quantified using BCA method. Lysed exosomal proteins were separated by SDS-PAGE and transferred onto a PVDF membrane. The PVDF membrane was then blocked with BSA at room temperature for $1 \mathrm{~h}$, followed by sequential incubation with primary antibody anti-CD63 or anti-TSG101, overnight and the respective HRP-conjugated secondary antibody. Signals were visualized on a gel imaging system (ChemiDoc XRS, BIO-RAD, USA).

\section{Extraction of exosomal RNA and reverse transcription}

Total RNA was extracted from plasma exosomes using the Multi-type Sample DNA/RNA Extraction-Purification Kit (Sansure Biotech Inc. Hunan, China), following the manufacturer's instructions. The RNA concentration was measured using the e-spect Spectrophotometer (Beijing labaid science and technology, Ltd., China). The OD $260 / 280 \mathrm{~nm}$ ratios of all RNA samples were $\geq 1.8$. A part of the RNA sample were used for reverse-transcription using the Mir-XTM miRNA first-strand synthesis kit (\#638313; TAKARA Bio Inc., USA), followed by qRT-PCR for miR-205. The remained RNA sample were stored at $-80^{\circ} \mathrm{C}$ and used for qRT-PCR of CA125, HE4 and TCF21.

\section{qPCR analysis for quantification of miR-205}

TB Green Advantage qPCR Premix (\#639676; TAKARA Bio Inc., USA) was used for qPCR on a 7500 Real-Time PCR System (Applied Biosystems, Thermo Fisher Scientific. Inc., USA). Briefly, two microliters of the cDNA products were used for as PCR template, and the final volume was $25 \mu \mathrm{l}$. U6 RNA was used as the internal reference gene, and the reaction conditions were as follows: $95^{\circ} \mathrm{C}$ pre-denaturation for $10 \mathrm{~s}, 95^{\circ} \mathrm{C}$ denaturation for $5 \mathrm{~s}$, and $60^{\circ} \mathrm{C}$ annealing for $20 \mathrm{~s}$, a total of 40 cycles to get dissociation curve. The relative expression level of exosomal miR-205 was analyzed using the $2^{-\Delta \Delta C t}$ relative quantitation method [10].

\section{qRT-PCR quantification of CA125, HE4 and TCF21}

The expression mRNA levels of CA125, HE4 and TCF21 were determined using One Step TB Green ${ }^{\circledR}$ PrimeScript ${ }^{\mathrm{TM}}$ Plus RT-PCR Kit (\#RR096A, TAKARA Bio Inc., USA) on the 7500 Real-Time PCR System. $\beta$-actin gene was used as an internal control. The reaction conditions were as follows: reverse transcription of RNA into cDNA at $42^{\circ} \mathrm{C}$ for $5 \mathrm{~min}, 95^{\circ} \mathrm{C}$ for 10 s, denaturation at $95^{\circ} \mathrm{C}$ for $5 \mathrm{~s}$, annealing at $60^{\circ} \mathrm{C}$ for $34 \mathrm{~s}$, and a total of 40 cycles. The relative expression of genes was calculated using the $2^{-\triangle \triangle \mathrm{CT}}$ method.

The primers used in this study were purchased from Shanghai Biotech Co., Ltd. China, and the primer sequences are listed in Table 1.

\section{Statistical analysis}

All data were analyzed using the SPSS 26.0 statistical software (IBM Corp, USA) and plotted by GraphPad Prism 7.0 (GraphPad Software Inc., USA). All data were expressed as mean \pm standard deviation or median

Table 1 Primer sequences for gene expression evaluation using qRT-PCR

\begin{tabular}{lll}
\hline Genes & Forward Sequence & Reverse Sequence \\
\hline CA125 & 5'-ACT GCC ACT GAG CCA ACA AGT TC-3' & 5'-GAC TGT GCC AAG ACT ATC CGA AGC-3' \\
HE4 & 5'-TCA ACA GAA GGA GGC AAT GTA T-3' & 5'-CAG CTG CTT AAT CTT ATG CTC G-3' \\
TCF-21 & 5'-CAG CGA TGT GGA GGA CCTTCA AG-3' & 5'-TCT CCT CGG TGC TCT CGT TGG-3' \\
B-ACtin & 5'-CTC CAT CCT GGC CTC GCT GT-3' & 5'-GCT GTC ACC TTC ACC GTT CC-3' \\
miR-205 & 5'-TCCTTC ATT CCA CCG GAG TCT G-3' & MRQ 3' Primer (provided in the kit) \\
U6 & 5'-GGA ACG ATA CAG AGA AGA TTA GC-3' & 5'-TGG AAC GCT TCA CGA ATT TGC G-3' \\
\hline
\end{tabular}


(interquartile range), according to data distribution. The Mann-Whitney $U$ test was used to compare the two groups, and the Kruskal-Wallis $H$ test or one-way ANOVA was used for comparison between multiple groups. The area under the curve (AUC) of the receiver operating characteristic curve (ROC) was used to analyze the diagnostic value of plasma exosomal miR-205, CA125, HE4 and TCF21 for OC. $P<0.05$ was considered statistically significant.

\section{Results}

Characteristics of plasma exosomes

We analyzed extracted exosomes with by three methods, TEM, NTA and WB. As shown in Fig. 1a, the extracted exosomes observed under TEM were cup-shaped or dishshaped vesicles with a diameter of about $100 \mathrm{~nm}$. WB results showed that the lysates of isolated plasma particles of all groups expressed the exosome-specific marker proteins, CD63 and TSG101 (Fig. 1b). The NTA results showed

\section{a}

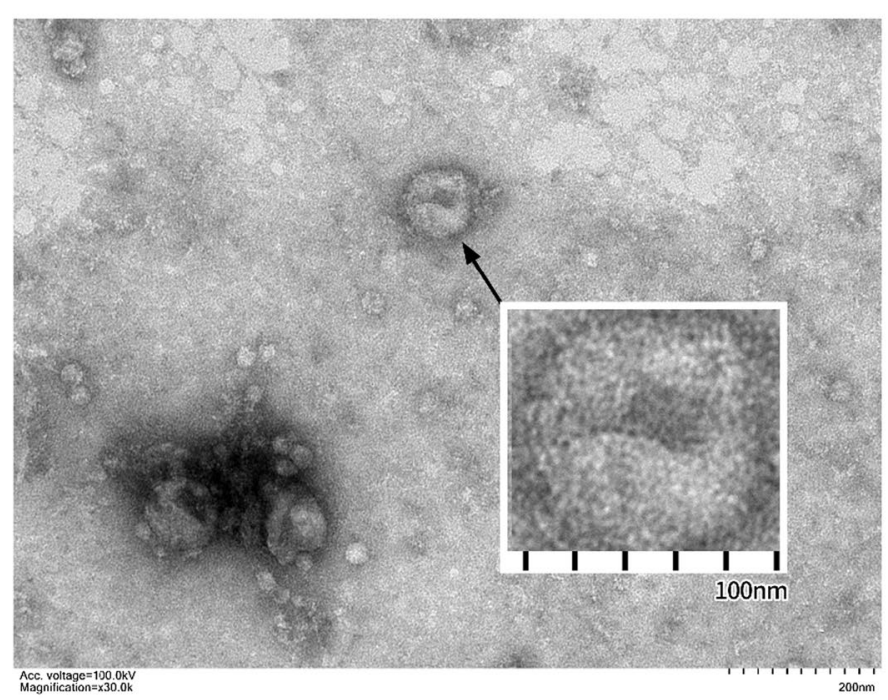

\section{b}

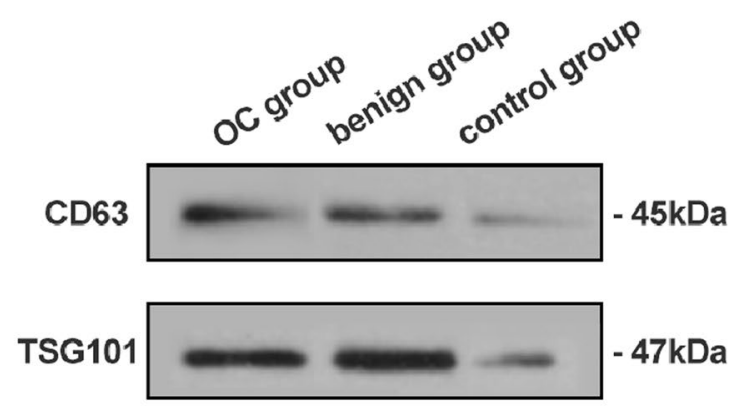

C

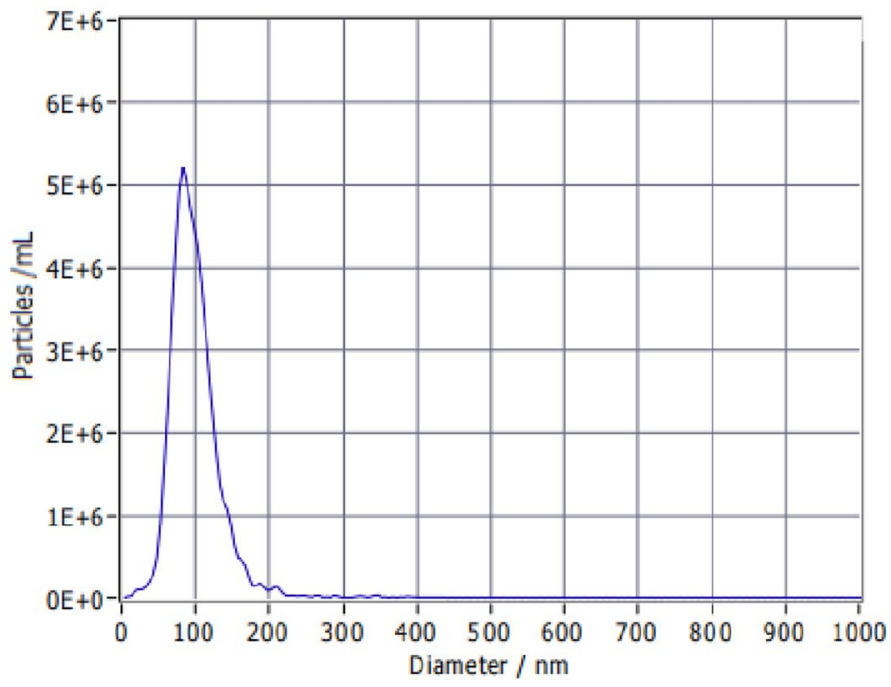

Fig. 1 Plasma exosome characteristics. a Transmission electron microscopy (TEM) revealed the round shape of exosomes. The bar represents $200 \mathrm{~nm}$. b. The marker proteins of exosomes (CD63 and TSG101) were detected by western blotting (WB). c. The Nanoparticle tracking analysis (NTA) presents the size and concentration distribution 
that the exosomes were distributed as particles with a diameter ranged from 20.7 to $345.2 \mathrm{~nm}$, with an average size of $98.6 \mathrm{~nm}$. And the total concentration of the exosomes was determined at $1.3 \mathrm{E}+12$ particles $/ \mathrm{mL}$ (Fig. 1c).

\section{Comparison of expression levels of plasma exosomal miR-205, CA125, HE4 and TCF21 among the three groups of patients and control}

The expression levels of the four RNAs, $m i R-205, C A 125$, HE4 and TCF21 in the three groups of OC patients and controls were determined by RT-qPCR as described in the Methods, and the results are shown in Table 2 and Fig. 2. We found that expression level of plasma exosomal miR-205 in the OC group was significantly higher than those in the benign and control groups $(P<0.01)$ (Fig. 2a). Expression level of plasma exosomes $C A 125$ in the OC group was the highest among the three groups, which was significantly different from the benign group $(P<0.01)$ (Fig. 2b). The levels of plasma exosomal HE4 in benign group was significantly lower than those in the $\mathrm{OC}$ and control groups $(P<0.01)$ (Fig. $2 \mathrm{c}$ ), whereas the expression level of plasma exosomal TCF21 in the OC group and the benign group was significantly lower than that of the control group $(P<0.05)$ (Fig. $2 \mathrm{~d})$. In addition, there was no significant difference in the expression levels of the four RNAs among the other groups that were not mentioned. $(P>0.05)$.

The relationship between the levels of the detected genes and the clinicopathological parameters of $O C$ patients

In $\mathrm{OC}$ patients, we detected the expression levels of exosomal genes, miR-205, CA125, HE4 and TCF21 and the protein concentrations of CA125 and HE4 in plasma samples. Together with the analysis of the patient's clinicopathological parameters, we found that only the expression level of plasma exosomes miR-205 in stage III-IV was higher than that in stage I-II $[4.59(2.23,9.39)$ vs $(1.28(0.65,3.35)]$. The level of miR-205 in the group with lymph node metastasis was higher than the group without lymph node metastasis $[5.15(2.40,10.02)$ vs $1.69(0.78,3.35)]$, and both were statistically significant $(P<0.05)$. All other analyses suggested there were correlations between the levels of the detected genes in plasma exosomes with age, menopausal status, FIGO stage, lymph node metastasis, and tumor site $(P>0.05)$, as shown in Table 3 .

Evaluate the diagnostic value of the four genes and protein CA125 and HE4 of plasma exosomes in OC

ROC curves were used to further evaluate the diagnostic efficacy of plasma exosomal genes miR-205, CA125, HE4, TCF21 and plasma exsosomal proteins CA125 and HE4 concentration for $\mathrm{OC}$, using the control group as a reference (Table 4 and Fig. 3). The AUC of plasma exosomal miR-205 was 0.715 (95\% CI: 0.590-0.841, $P=0.002)$, with a sensitivity of $66.7 \%$ and a specificity of $78.1 \%$. The AUC of plasma exosomal CA125, HE4, and TCF21 were 0.642, 0.554 , and 0.673 , respectively, which were all less than 0.7 , suggesting they are not ideal for diagnosis of OC. The AUC of protein CA125 was 0.915 (95\% CI: 0.846-0.983, $P<0.001$ ), with a sensitivity of $74.3 \%$ and a specificity of 93.7\%, and the AUC of protein HE4 was 0.779 (95\% CI: $0.668-0.889, P<0.001)$, with a sensitivity of $55.6 \%$ and a specificity of $100 \%$. The combined index analysis showed that exosomal miR-205 together with protein CA125 or HE4, the diagnostic AUC was 0.930 (95\% CI: 0.865-0.995, $P<0.0001$ ) or 0.827 (95\% CI: 0.726-0.929, $P<0.0001)$. The AUC of the triple gene diagnosis was increased to 0.951 (95\% CI: 0.899-1.004, $P<0.0001$ ), and the sensitivity and the specificity was increased to 100 and $86.1 \%$, respectively. However, the AUC of traditional plasma proteins, CA125 and HE4, was 0.939 (95\% CI: $0.883-0.995, P<0.0001)$, the sensitivity was $96.9 \%$, and the specificity was $83.3 \%$.

\section{Discussion}

$\mathrm{OC}$ is the fifth most common tumor that threatens the health of women worldwide, and its mortality rate ranks the first among gynecological tumors [11].

Table 2 Relative expression levels of plasma exosomal miR-205, CA125, HE4 and TCF21 in OC, benign and control groups [Median(Q1, Q3)]

\begin{tabular}{llll}
\hline Tested marker gene & OC group $(\boldsymbol{n}=\mathbf{3 6})$ & Benign group $(\boldsymbol{n}=\mathbf{3 1})$ & Control group $(\boldsymbol{n}=\mathbf{3 2})$ \\
\hline miR-205 & $3.24(1.56,8.48)^{\mathrm{ab}}$ & $1.93(0.67,3.30)$ & $1.34(1,2.41)$ \\
CA125 & $1.97(0.87,3.25)^{\mathrm{a}}$ & $0.66(0.15,1.79)$ & $1(1,1.56)$ \\
HE4 & $1.57(0.79,2.23)^{\mathrm{a}}$ & $0.42(0.13,1.19)^{\mathrm{b}}$ & $1(1,1.16)$ \\
TCF21 & $0.94(0.51,1.71)^{\mathrm{b}}$ & $0.85(0.15,2.04)^{\mathrm{b}}$ & $1(1,2.47)$ \\
\hline
\end{tabular}

${ }^{a}$ indicates that there is a significant difference compared with the benign group $(P<0.05)$

${ }^{b}$ indicates that there is a significant difference compared with the control group $(P<0.05)$

$P$ values were calculated by the Kruskal-Wallis $\mathrm{H}$ test 


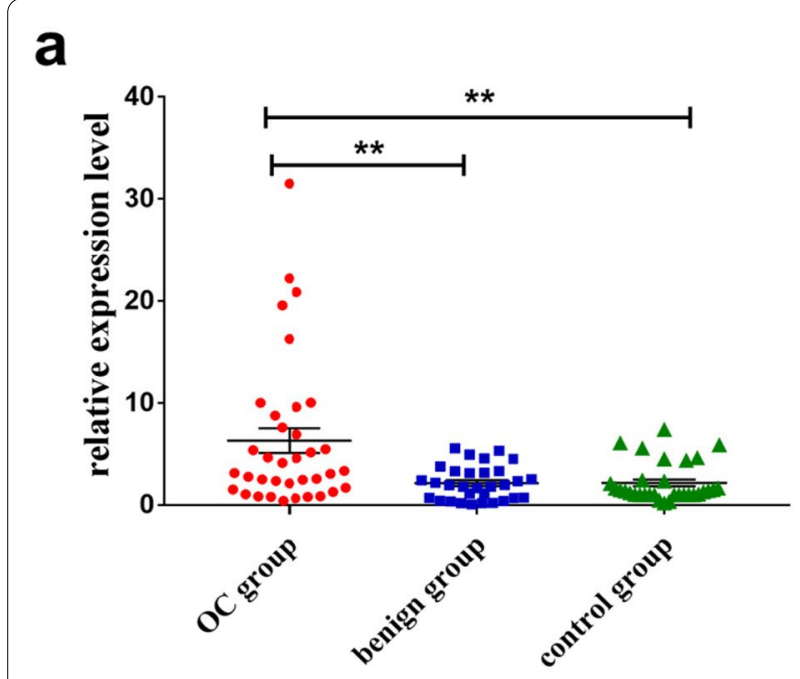

Exosomal miR-205

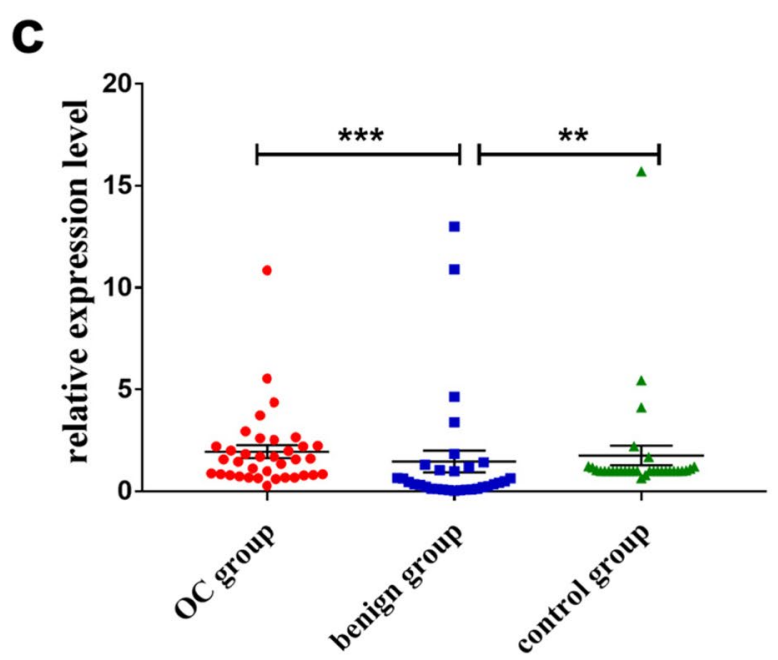

Exosomal HE4

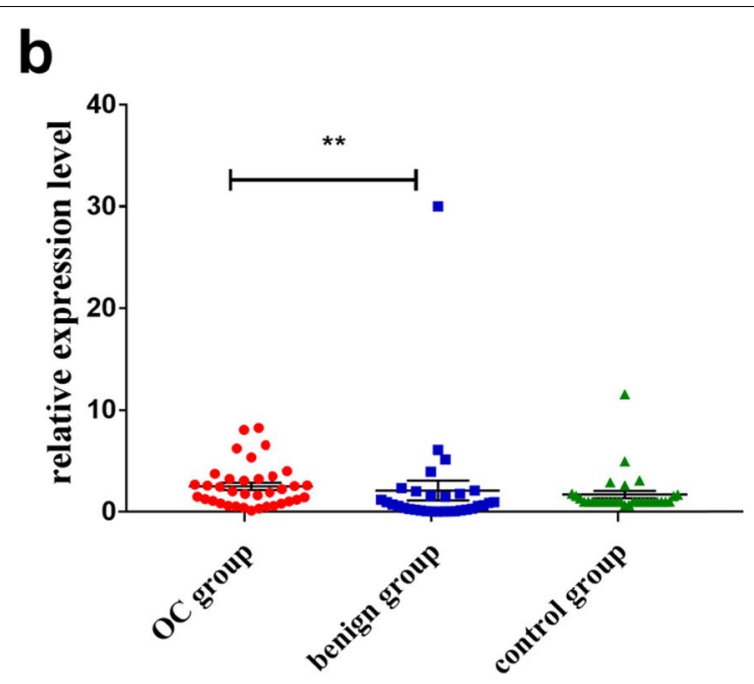

Exosomal CA125

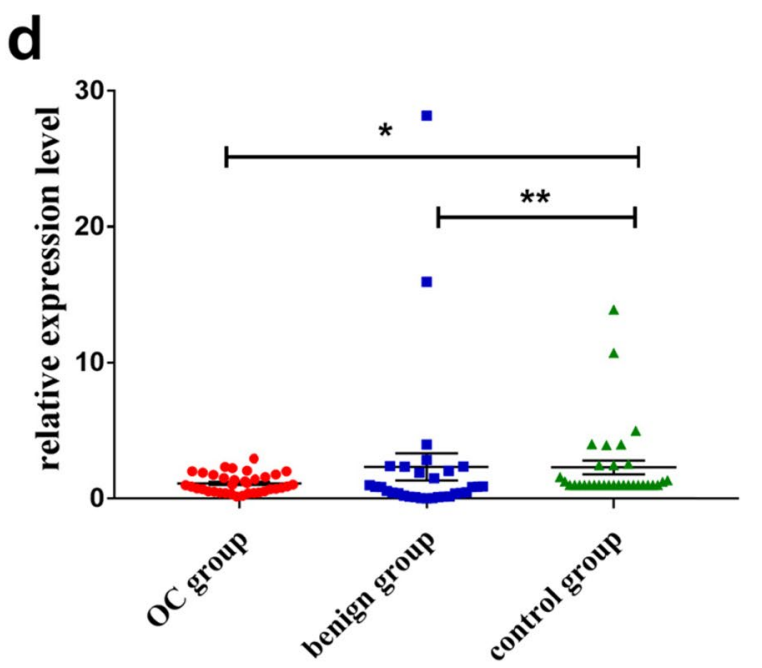

Exosomal TCF21

Fig. 2 The expression levels of plasma exosomal miR-205, CA125, HE4 and TCF21 among three groups of people (Kruskal-Wallis $H$ test). ${ }^{*} p<0.05$; ${ }^{* *} p<0.01 ;{ }^{* *} p<0.001$

According to the latest cancer report in 2021, the incidence of OC accounts for about $3.4 \%$ of all tumors, but the mortality rate is nearly $5 \%$, which means that there are approximately 310,000 new cases and 200,000 deaths in 2020 [1]. Early detection and early diagnosis are the key to improving the prognosis of OC patients. However, studies have shown that the conventional screening method of serum CA125 combined with vaginal ultrasound cannot reduce the mortality of patients $[12,13]$. At present, there are no effective screening methods for $\mathrm{OC}$, and the diagnosis must rely on the pathological results of tissue biopsy, which brings great pain to patients [14]. The exosomes-based liquid biopsy technology can probe disease progressions through non-invasive or minimally invasive sampling. In recent years, it has received more and more attention in tumor screening, diagnosis and treatment, and prognosis evaluation [15]. This also provides new ideas for OC screening and early diagnosis.

In this study, we extracted exosomes in plasma samples of all subjects with commercial reagents, and then used three methods (TEM, WB, and NTA) to analyze the 
Table 3 Relationship between the detected biomarkers and clinicopathological parameters in OC patients

\begin{tabular}{|c|c|c|c|c|c|}
\hline Tested biomarkers & $\begin{array}{l}\text { Age }(<50 \text { years } \\
\text { vs. } \geq 50 \text { years })\end{array}$ & $\begin{array}{l}\text { Menopause (Yes } \\
\text { vs. No) }\end{array}$ & $\begin{array}{l}\text { Metastases lymph } \\
\text { nodes (Yes vs. No) }\end{array}$ & $\begin{array}{l}\text { FIGO stage (stage I+ II vs. } \\
\text { stage III + IV) }\end{array}$ & $\begin{array}{l}\text { Tumor site } \\
\text { (unilateral vs. } \\
\text { bilateral }\end{array}$ \\
\hline & Pvalue & & & & \\
\hline exosomal miR-205 & 0.689 & 0.768 & $0.007^{*}$ & $0.032^{*}$ & 0.553 \\
\hline exosomal CA125 & 0.849 & 0.664 & 0.292 & 0.845 & 0.761 \\
\hline exosomal HE4 & 0.689 & 0.520 & 0.435 & 0.192 & 0.665 \\
\hline exosomal TCF21 & 0.337 & 0.286 & 0.588 & 0.557 & 0.911 \\
\hline protein $\mathrm{CA} 125(\mathrm{U} / \mathrm{mL})$ & 0.614 & 0.271 & 0.830 & 0.614 & 0.665 \\
\hline protein HE4 (pmol/L) & 0.374 & 0.286 & 0.934 & 0.362 & 0.170 \\
\hline
\end{tabular}

$P$ values were calculated by Mann-Whitney $U$ test. ${ }^{*} P<0.05$

Table 4 Diagnostic value of single detected biomarker and combined detections for OC

\begin{tabular}{|c|c|c|c|c|c|c|}
\hline Test biomarkers & AUC & $95 \% \mathrm{Cl}$ & $P$ & Sensitivity\% & Specificity\% & Youden's index \\
\hline protein CA125 (U/mL) & 0.915 & $0.846-0.983$ & $<0.001$ & 74.3 & 93.7 & 0.68 \\
\hline protein HE4 (pmol/L) & 0.779 & $0.668-0.889$ & $<0.001$ & 55.6 & 100.0 & 0.56 \\
\hline exosomal miR205 & 0.715 & $0.590-0.841$ & 0.002 & 66.7 & 78.1 & 0.45 \\
\hline exosomal CA125 & 0.642 & $0.501-0.784$ & 0.044 & 75.0 & 68.7 & 0.44 \\
\hline exosomal HE4 & 0.554 & $0.403-0.704$ & 0.446 & 58.3 & 84.4 & 0.43 \\
\hline exosomal TCF21 & 0.673 & $0.539-0.808$ & 0.014 & 47.2 & 59.4 & 0.07 \\
\hline protein $\mathrm{CA} 125$ + protein HE4 & 0.939 & $0.883-0.995$ & $<0.0001$ & 96.9 & 83.3 & 0.80 \\
\hline protein CA125 + exosomal miR-205 & 0.930 & $0.865-0.995$ & $<0.0001$ & 96.9 & 83.3 & 0.80 \\
\hline protein HE4 + exosomal miR-205 & 0.827 & $0.726-0.929$ & $<0.0001$ & 96.9 & 69.4 & 0.66 \\
\hline $\begin{array}{l}\text { protein CA } 125 \text { + protein HE4 + exosomal } \\
\text { miR-205 }\end{array}$ & 0.951 & $0.899-1.004$ & $<0.0001$ & 100 & 86.1 & 0.86 \\
\hline
\end{tabular}

extracted exosomes. They were visually observed under TEM. The particles were in the form of cup-shaped vesicles with a diameter of about $100 \mathrm{~nm}$. WB results showed that the particles expressed exosomal characteristic proteins CD63 and TSG101. NTA results showed that the average size of the particles was $98.6 \mathrm{~nm}$ and contained a high concentration $(1.3 \mathrm{E}+12$ particles $/ \mathrm{mL})$. These results were consistent with the characteristics of exosomes as previously reported [16], and confirmed the success in extraction of plasma exosomes. Exosomes are involved in the communication between cells, and they are widely

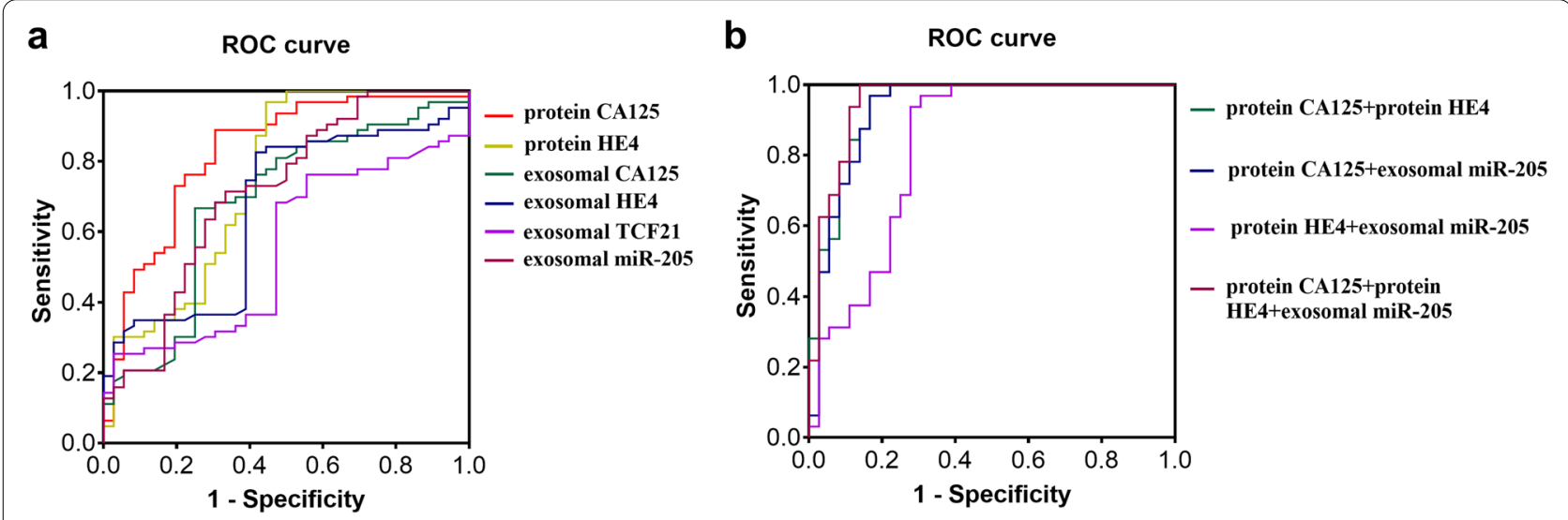

Fig. 3 Receiver operator characteristics (ROC) curves for prediction of OC by the levels of plasma exosomal miR-205 and CA125, HE4 and TCF21 mRNAs alone or combined with traditional biomarkers, CA125 and HE4 proteins 
present in biological cells and various body fluids [17]. Exosomes derived from cancer cells in the early stages of tumors can be released into the blood in large quantities and are highly stable in peripheral blood, making it possible to be used as new tumor markers. A number of studies have shown that exosomes play an important role in the occurrence, development and drug resistance of ovarian cancer, and therefore exosomes have the capability to guide the diagnosis, treatment and prognosis evaluation of OC [18-21].

We further analyzed the expression levels of the four genes miR-205, CA125, HE4 and TCF21 in plasma exosomes and the concentrations of plasma proteins, CA125 and HE4. We found that the expression level of plasma exosomal miR-205 in the OC group were significantly higher than that in the benign and control groups, which was consistent with our previous results in OC tissues and cell lines [6]. At the same time, together with the analysis of the clinicopathological parameters of OC patients, we found that the expression level of plasma exosomal miR-205 in OC patients was higher in advancedstage (stage III-IV) than in early-stage (stage I-II), the group with lymph node metastasis was higher than the group without lymph node metastasis, and the differences were statistically significant. However, the traditional serum markers of plasma proteins, CA125 and HE4, were not related to the FIGO staging of OC and lymph node metastasis. Compared with traditional markers, plasma exosomal miR-205 performs better in early diagnosis and prognostic evaluation of OC. Thus, it is expected that exosomal miR-205 will become a novel tumor biomarker to assist in the screening and early diagnosis of OC. We previously have shown that miR-205 was highly expressed in OC tissues, and miR-205 mimic in cell lines promotes the invasion and metastasis of OC cells. We highly suspect that the high expression of miR-205 in OC tissues may transmit relevant signals through exosomes, thereby accelerating the overall invasion and metastasis process of OC. All these indicate that plasma exosomal miR-205 becomes an early diagnostic biomarker for $\mathrm{OC}$ and can be used for prognostic evaluation of OC.

MiR-205 is one of the highly conservative miRNAs. It has a dual effect as both a tumor promotion factor and a tumor suppressor [22]. Studies have found that the expression of miR-205 was reduced in sera of breast cancer patients [23], but it was up-regulated in OC tissues and cells and related to the growth and metastasis of OC [24], which was consistent with our previous published results [6]. In addition, it has been suggested that vascular endothelial growth factor (VEGF) upregulates miR-205 expression, which leads to the downregulation of Ezrin and Lamin A/C and, thereby, promotes the invasion of OC cells [25]. miR-205 has been shown to regulate the proliferation and invasion of $\mathrm{OC}$ cells by inhibiting the expression of PTEN/Smad4 [26]. Other studies have shown that miR-205 directly negatively regulates ZEB1 to promote the clinical progress of EOC patients [27]. A recent study also showed that miR-205 derived from OC cell exosomes promoted the transformation of OC by inducing angiogenesis [28]. Therefore, together with the results obtained from our current study, these studies confirmed that miR-205 had abnormal expression in OC and was related to the invasion of $\mathrm{OC}$.

We further evaluated the diagnostic value of plasma exosomal miR-205 for OC, together with the current traditional biomarkers, plasma proteins CA125 and HE4. Through ROC curve analysis, it was found that the AUC of plasma proteins CA125 and HE4 were 0.915 and 0.779 , respectively, the sensitivity was 74.3 and $55.6 \%$, and the specificity was 93.7 and $100 \%$, respectively. This result confirmed that although the traditional biomarkers provide valuable diagnosis of $\mathrm{OC}$, they are less sensitive, resulting in missing diagnoses. The plasma exosomal miR-205 alone diagnosed OC with an AUC of 0.715 , a sensitivity of $66.7 \%$, and a specificity of $78.1 \%$. However, when combined with the two traditional markers, plasma exosomal miR-205 significantly improved the early diagnosis of $\mathrm{OC}$, which increased the AUC of CA125 from 0.915 to 0.930 , and HE4 from 0.779 to 0.827. Moreover, combination of the detection of the three biomarkers increases the AUC to 0.951 , the sensitivity to $100 \%$, and the specificity to $86.1 \%$. Taken together, plasma exosomal miR-205 expression is of a high value in OC diagnoses, and when combined with the traditional biomarkers, CA125 and HE4 proteins, it effectively improves the diagnostic effectiveness of OC. Importantly, our study supports the detection of all the three biomarkers are, in particular, suitable for early screening of OC.

The study also found that the plasma exosomal CA125 and HE4 mRNA levels in the OC group were higher than those in the benign group, but there was no statistical difference from the control group. It was inconsistent with the findings by Fawzy et al. that the expression levels of CA125 and HE4 in OC tissues were higher than those of benign tumor tissues and normal ovarian tissues [29]. In addition, the expression level of plasma exosomal TCF21 in the OC and benign groups was lower than the control group, but there was no difference between the OC and benign groups. This was inconsistent with our previous results found in OC tissues [6]. The reason for this inconsistency is likely due to the inherent differences in gene expression patterns in plasma exosomes and tissues. Studies have shown that the differential expression profile of serum exosomal mRNA was not completely consistent with the tissue mRNA level [30], which explains the inconsistency to some points. On the other hands, it may also be due to the selective packaging of the mRNA molecules contained in exosomes, as 
exosomes regulate the packaging process of their internal RNA through a variety of ways [31]. In addition, the effects of differences in mRNA stability, sample source, sample size, and storage time may also account for the variation.

Although our study demonstrates the advantages of the use of plasma exosomal miR-205 as a new biomarker for OC, it has some limitations, for example, the small samples, which may lead to variations. In future studies, we will increase the sample size, preferably including cases from multiple hospitals. Nevertheless, the expression of miRNA had tissue and disease specificity [32, 33], and can be secreted in the form of exosomes and stably exists in the peripheral blood circulation [34]. It can also be delivered to the tumor microenvironment or distant organs through exosomes, and promotes tumor angiogenesis and metastasis by targeting gene expression in recipient cells [35]. We conclude that plasma exosomal miR-205 is a novel biomarker for OC, which is of a high reference value for early screening of OC.

\section{Conclusions}

In summary, our results showed that plasma exosomal miR-205 has an obvious merit in OC diagnosis, and when combined with the traditional serological tumor biomarkers, it improves the diagnostic efficiency of OC. In addition, the plasma exosomal miR-205 level is related to $\mathrm{OC}$ staging and lymph node metastasis, which provides a valued reference for the early diagnosis and prognostic evaluation of OC patients.

\section{Abbreviations \\ OC: Ovarian cancer; CA125: Cancer antigen 125; FIGO: International Federa- tion of Gynecology and Obstetrics; HE4: Human epididymis protein 4; ROC: Receiver operating characteristic curves; AUC: Area under the curve; $\mathrm{Cl}$ : Confidence interval; TCF21: Transcription factor 21.}

\section{Acknowledgements}

Not applicable.

\section{Authors' contributions}

Z-LH and C-ZJ conceived the study and designed the study methods and wrote the manuscript. Z-M and $Y-X$ contributed study materials from patients. Z-ZH, W-MY, C-YW and Z-CJ collected, assemble, and interpreted the data; L-YH and H-LQ performed statistical analysis. All authors read and approved the final version of the manuscript.

\section{Funding}

This research was supported by Zhejiang Medical and Health Science and Technology Program Fund (No. 2019KY131 to L. Zhang; No. 2019KY133 to Z. Chen) and Hangzhou Science and Technology Plan Development Project Fund (No. $20191203 B 106$ to L. Zhang; 20201203B206 to Z. Chen).

\section{Availability of data and materials}

The data supporting this study are all included in this article.

\section{Declarations}

Ethics approval and consent to participate

The study was approved by the Human Ethics Committee of the Affiliated Hospital of Hangzhou Normal University, and the ethics number was 2019 (Len 02)-HS-03.

\section{Consent for publication}

Yes.

Competing interests

The authors declare that they have no competing interests.

\section{Author details}

${ }^{1}$ The Medical School of Hangzhou Normal University, Hangzhou 310015, China. ${ }^{2}$ The Clinical laboratory of The Affiliated Hospital of Hangzhou Normal University, Hangzhou 310015, China. ${ }^{3}$ Institute of Basic Medicine and Cancer of The Cancer Hospital of the University of Chinese Academy of Sciences

(Zhejiang Cancer Hospital), Hangzhou 310022, China.

Received: 9 September 2021 Accepted: 3 February 2022

Published online: 19 February 2022

\section{References}

1. Sung H, Ferlay J, Siegel RL, Laversanne M, Soerjomataram I, Jemal A, et al. Global Cancer statistics 2020: GLOBOCAN estimates of incidence and mortality worldwide for 36 cancers in 185 countries. CA Cancer I Clin. 2021;71(3):209-49.

2. Moore RG, Bast RC Jr. How do you distinguish a malignant pelvic mass from a benign pelvic mass? Imaging, biomarkers, or none of the above. J Clin Oncol. 2007;25(27):4159-61.

3. Crosby D. Delivering on the promise of early detection with liquid biopsies. Br J Cancer. 2022;126(3):313-5.

4. Halvaei S, Daryani S, Eslami SZ, Samadi T, Jafarbeik-Iravani N, bakhshayesh TO, et al. Exosomes in cancer liquid biopsy: a focus on breast cancer. Mol Ther Nucleic Acids. 2018;10:131-41.

5. Macias M, Alegre E, Diaz-Lagares A, Patino A, Perez-Gracia JL, Sanmamed $\mathrm{M}$, et al. Liquid biopsy: from basic research to clinical practice. Adv Clin Chem. 2018:83:73-119.

6. Wei J, Zhang L, Li J, Zhu S, Tai M, Mason CW, et al. MicroRNA-205 promotes cell invasion by repressing TCF21 in human ovarian cancer. J Ovarian Res. 2017;10(1):33.

7. Ma Y, Wang X, Qiu C, Qin J, Wang K, Sun G, et al. Using protein microarray to identify and evaluate autoantibodies to tumor-associated antigens in ovarian cancer. Cancer Sci. 2021;112(2):537-49.

8. Mukama T, Fortner RT, Katzke V, Hynes LC, Petrera A, Hauck SM, et al. Prospective evaluation of 92 serum protein biomarkers for early detection of ovarian cancer. Br J Cancer. 2022. https://doi.org/10.1038/ s41416-021-01697-Z.

9. Zeppernick F, Meinhold-Heerlein I. The new FIGO staging system for ovarian, fallopian tube, and primary peritoneal cancer. Arch Gynecol Obstet. 2014;290(5):839-42.

10. Livak KJ, Schmittgen TD. Analysis of relative gene expression data using real-time quantitative PCR and the 2(-Delta Delta $C(T))$ method. Methods. 2001;25(4):402-8.

11. Torre LA, Trabert B, Desantis CE, Miller KD, Samimi G, Runowicz CD, et al. Ovarian cancer statistics, 2018. CA Cancer J Clin. 2018;68(4):284-96.

12. Buys SS, Partridge E, Black A, Johnson CC, Lamerato L, Isaacs C, et al. Effect of screening on ovarian cancer mortality: the prostate, lung, colorectal and ovarian (PLCO) cancer screening randomized controlled trial. JAMA. 2011;305(22):2295-303.

13. Pinsky PF, Yu K, Kramer BS, Black A, Buys SS, Partridge E, et al. Extended mortality results for ovarian cancer screening in the PLCO trial with median 15years follow-up. Gynecol Oncol. 2016;143(2):270-5.

14. Williams RM, Lee C, Galassi TV, Harvey JD, Leicher R, Sirenko M, et al. Noninvasive ovarian cancer biomarker detection via an optical nanosensor implant. Sci Adv. 2018;4(4):eaaq1090. 
15. Bunda S, Zuccato JA, Voisin MR, Wang JZ, Nassiri F, Patil V, et al. Liquid biomarkers for improved diagnosis and classification of CNS tumors. Int J Mol Sci. 2021;22(9):4548.https://doi.org/10.3390/ijms22094548.

16. Pegtel DM, Gould SJ. Exosomes. Annu Rev Biochem. 2019;88:487-514.

17. ALENQUER M, AMORIM MJ. EXosome biogenesis, regulation, and function in viral infection. Viruses. 2015;7(9):5066-83.

18. Lutgendorf SK, Thaker PH, Arevalo JM, Goodheart MJ, Slavich GM, Sood AK, et al. Biobehavioral modulation of the exosome transcriptome in ovarian carcinoma. Cancer. 2018:124(3):580-6.

19. Shen J, Zhu X, Fei J, Shi P, Yu S, Zhou J. Advances of exosome in the development of ovarian cancer and its diagnostic and therapeutic prospect. Onco Targets Ther. 2018;11:2831-41.

20. Qian L, Yang X, Li S, Zhao H, Gao Y, Zhao S, et al. Reduced O-GlcNAcylation of SNAP-23 promotes cisplatin resistance by inducing exosome secretion in ovarian cancer. Cell Death Discov. 2021;7(1):112.

21. Li X, Liu Y, Zheng S, Zhang T, Wu J, Sun Y, et al. Role of exosomes in the immune microenvironment of ovarian cancer. Oncol Lett. 2021;21(5):377.

22. Chauhan N, Dhasmana A, Jaggi M, Chauhan SC, Yallapu MM. miR-205: a potential biomedicine for cancer therapy. Cells. 2020;9(9):1957. https:// doi.org/10.3390/cells9091957.

23. Zhang $\mathrm{H}$, Li B, Zhao H, Jin C. The expression and clinical significance of serum miR-205 for breast cancer and its role in detection of human cancers. Int J Clin Exp Med. 2015;8(2):3034.

24. Li J, Hu K, Gong G, Zhu D, Wang Y, Liu H, et al. Upregulation of MiR-205 transcriptionally suppresses SMAD4 and PTEN and contributes to human ovarian cancer progression. Sci Rep. 2017;7:41330.

25. Li J, Li L, Li Z, Gong G, Chen P, Liu H, et al. The role of miR-205 in the VEGFmediated promotion of human ovarian cancer cell invasion. Gynecol Oncol. 2015;137(1):125-33.

26. Chu P, Liang A, Jiang A, Zong L. miR-205 regulates the proliferation and invasion of ovarian cancer cells via suppressing PTEN/SMAD4 expression. Oncol Lett. 2018;15(5):7571-8.

27. Niu K, Shen W, Zhang Y, Zhao Y, Lu Y. MiR-205 promotes motility of ovarian cancer cells via targeting ZEB1. Gene. 2015;574(2):330-6.

28. He L, Zhu W, Chen Q, Yuan Y, Wang Y, Wang J, et al. Ovarian cancer cellsecreted exosomal miR-205 promotes metastasis by inducing angiogenesis. Theranostics. 2019;9(26):8206-20.

29. Fawzy A, Mohamed MR, Ali MA, Abd El-Magied MH, Helal AM. Tissue CA125 and HE4 gene expression levels offer superior accuracy in discriminating benign from malignant pelvic masses. Asian Pac J Cancer Prev. 2016;17(1):323-33.

30. Ji J, Chen R, Zhao L, Xu Y, Cao Z, Xu H, et al. Circulating exosomal mRNA profiling identifies novel signatures for the detection of prostate cancer. Mol Cancer. 2021;20(1):58

31. Janas T, Janas MM, Sapon K, Janas T. Mechanisms of RNA loading into exosomes. FEBS Lett. 2015;589(13):1391-8.

32. Liu H, Wang G, Huang Y, Zhao C, Chen J, Wang X. Identification specific miRNA in $\mathrm{t}(4 ; 14)$ multiple myeloma based on miRNA-mRNA expressing profile correlation analysis. J Cell Biochem. 2018. https://doi.org/10.1002/ jcb.27537.

33. Usuba W, Urabe F, Yamamoto Y, Matsuzaki J, Sasaki H, Ichikawa M, et al. Circulating miRNA panels for specific and early detection in bladder cancer. Cancer Sci. 2019;110(1):408-19.

34. Madhavan B, Yue S, Galli U, Rana S, Gross W, Müller M, et al. Combined evaluation of a panel of protein and miRNA serum-exosome biomarkers for pancreatic cancer diagnosis increases sensitivity and specificity. Int J Cancer. 2015;136(11):2616-27.

35. Tkach M, Thery C. Communication by extracellular vesicles: where we are and where we need to go. Cell. 2016;164(6):1226-32.

\section{Publisher's Note}

Springer Nature remains neutral with regard to jurisdictional claims in published maps and institutional affiliations. 